\title{
Fundamental Studies on Rankine Source Panel Method Fully Based on B-splines
}

\author{
by Nikolay E. Markov*, Member Kazuo Suzuki*, Member
}

\begin{abstract}
Summary
A free-surface boundary element method is presented for solving three-dimensional steady ship wave problem. The method approximates geometry and potential with a higher degree of continuity, compared to the other Rankine source methods. Global parametric B-spline tensor products define both the ship hull and the free-surface geometry. A skinning type algorithm based on de Boor procedure is developed to perform the surface fitting. B-splines of various degrees represent the unknown singularity distribution. The functions describing geometry and potential have independent structures and are connected on their parametric space. The influence coefficients are evaluated by adaptive Gauss quadratures after removing the singularity of Rankine source analytically. Kelvin's type free-surface condition and Kutta-like radiation condition ensure unique solutions. Involved derivatives of the potential are obtained directly, without using a difference scheme. Computations of the wave resistance for Wigley, Series 60, HTC container ship and SR221B tanker hull forms, approximated by a single patch, demonstrate the accuracy of the method.
\end{abstract}

\section{Introduction}

Rankine source panel method ${ }^{1)}$ is one of the popular mathematical models for solving ship wave resistance problem nowadays. The method provides a numerical solution for the integral equation describing the fluid disturbance caused by ships. It implements the boundary element technique for the ship hull surface and some free-surface area near the ship. Naval architects use the Rankine source method mainly to reduce the time and cost of ship hull form optimization.

The most commonly encountered boundary elements in practice are the flat panels and some other panels, which approximate the ship hull geometry locally. Although these panels describe the geometry relatively easy, they do not give an easy way to change the hull smoothly in the optimization process. The global Bspline geometry description proposed here provides a more sophisticated manipulation of the hull form. When using the new geometry, we know what to change and how much to change it, without losing the ship-like form of the hull topologically. In addition the related higher-order approximation of the solution detects even the smallest geometric changes well, and the gradients of the wave resistance can be calculated accurately. Therefore we introduce a higher-order method fully

\section{* Yokohama National University}

Received 27th Dec. 1999

Read at the Spring meeting 18, 19th May 2000 based on $\mathrm{B}^{-}$-splines in this paper.

Since the fluid viscosity is eliminated in the steady potential problem, the solution of the governing integral equation is not unique. It allows the waves, created by ship motion, to spread in the upstream direction. A so -called radiation condition is imposed to ensure uniqueness. The radiation condition has been achieved so far by use of upstream difference formulae ${ }^{1)}$, by shifting the collocation points ${ }^{2 \sim 6)}$, or by applying Kutta-like condition $^{7 \sim 8)}$. The last one can be implemented in a higher-order panel method, and we will use it below.

Sclavounos and Nakos ${ }^{7}$ used a bi-quadratic spline scheme to approximate the solution of the integral equation. They discretized the boundary surfaces by plane quadrilateral panels, which could be classified as a lower-order panel method. We will try to increase the accuracy, combining the higher-order of potential approximation with a higher-order of geometry description. Besides, in order to make the method work, we have to perform the integration of the singularities numerically. It gives us an additional freedom to use any degree of approximation. We also compare solutions with and without spline end conditions, with one or more collocation points per panel later in this paper. When using end conditions we choose the more neutral physically "not-a-knot" conditions in comparison with the natural ones, adopted by Sclavounos and Nakos.

Another $\mathrm{B}^{-}$spline based panel method has been developed over the last decade. Hsin et $\mathrm{a}^{9)}$ introduced a two -dimensional method based on $\mathrm{B}^{-}$-splines. Maniar ${ }^{10)}$ and 
Lee et $\mathrm{al}^{11 \sim 12)}$ created the three-dimensional case. Since it is a Kelvin-Havelock source method, its governing integral equation is applied on the rigid surfaces only. The practical applications of this method are directed mainly to the free- ${ }^{-}$surface flows, surrounding very large floating structures. The present work extends the above technique to a Rankine source method for ships by discretizing some part of the free-surface area.

The advantages for developing higher-order panel method based on $\mathrm{B}$-splines are: The more sophisticated B-spline geometry will allow a simpler treatment of the ship hull optimization problem in the future. The method can be switched to any degree of approximation, i.e. any degree of continuity of the solution can be achieved and the short-scale wavelengths can be evaluated easier. The radiation condition does not depend on a choice of the upstream difference formulae. $\mathrm{B}^{-}$spline scheme is free of numerical damping ${ }^{7)}$. The derivatives of the potential on the body and on the free -surface are calculated analytically. Standard CAD/ CAM systems provide input and output of the geometric data in B-spline format directly.

Only two $\mathrm{B}^{-}$-spline patches are used in the description of the problem. One of those approximates the ship hull, and the other one approximates the free-surface domain. In consequence, the geometric quantities, such as normal vector and curvature, and the hydrodynamic quantities, such as velocity field and pressure, are naturally continuous over the entire ship hull surface, with the only exception-its boundary line. Therefore, although the present technique still remains a boundary element method, its discrete panel nature could be questioned. We should note also that the $\mathrm{B}^{-}$-spline patches, describing geometry and potential, possess different structures (different number, degree and parameterization of their polynomials). Such independent structures allow the use of different refinement of geometry and potential on the same place of the hull surface. Thus the choice of potential approximation depends on the convergence only, whereas the choice of geometry approximation depends on the specific ship hull form.

The calculated wave patterns show satisfactory accuracy and smoothness of the numerical solution. The results for the wave elevation and the wave resistance coefficient are in agreement with the experimental data, except for the resistance coefficient of the tanker. The numerical results are compared with the results of a lower-order panel method as well.

\section{B-splines in brief}

Spline functions are powerful tool for practical approximation of surfaces. B-spline represents a special case of spline function ${ }^{13)}$. First, its coefficients have geometric sense. One can comprehend intuitively what will happen to the $\mathrm{B}$-spline by changing one or more of its coefficients. That makes B-spline very suitable for surface design and optimization. Secondly, $\mathrm{B}^{-}$splines and their derivatives possess basis, so that the coefficients of the $\mathrm{B}$-splines can be considered as coordinates in a functional space. The basis function of any degree can be built by the basis functions of the lower degrees. This automatic generation ensures some desirable degree of continuity. For instance, for a $\mathrm{B}^{-}$spline of $3^{\text {rd }}$ degree, its second derivatives could be constructed to be continuous everywhere.

Consider a set of data points $\boldsymbol{r}_{1}, \cdots, \boldsymbol{r}_{l}, \boldsymbol{r}_{i}=\left(x_{i}, y_{i}, z_{i}\right)$ in space and corresponding parameter values (or knots or breakpoints) $u_{1}, \cdots, u_{L}$. Then the three-dimensional Bspline of $\mathrm{N}$-th degree is a curve $\boldsymbol{r}=\boldsymbol{r}(u)$, determined by the same knots and unknown control vertices (coefficients) $\boldsymbol{d}_{1}, \cdots, \boldsymbol{d}_{L+N-1}, \boldsymbol{d}_{i}=\left(X_{i}, Y_{i}, Z_{i}\right)$, such that $\boldsymbol{r}\left(u_{i}\right)=\boldsymbol{r}_{i}, i=1, \cdots, L$; in other words, such that interpolates to the data points ${ }^{13}$. The $\mathrm{B}^{-}$spline form of this piecewise polynomial parametric curve is

$$
\boldsymbol{r}(u)=\sum_{i=1}^{L+N-1} \boldsymbol{d}_{i} B_{i}^{N}(u)
$$

Figure 1( a ) - ( b ) illustrates the geometric dependence of B-spline curve on its coefficients $\boldsymbol{d}_{i}, i=1, \cdots, L+N-1$. Here, the dotted line connects the coefficients and the continuous line represents the $\mathrm{B}$-spline curve. One can see how the curve (imagine for example a ship waterline) changes after shifting the coefficient $\boldsymbol{d}_{i}$ in Figure 1(b). The functions $B_{i}^{N}(u), i=1 \cdots L+N-1$ are basis of the $\mathrm{B}$-spline of $\mathrm{N}$-th degree. We derived the polynomial formula ( 2 ), which allows constructing the basis about three times faster than the commonly used recursive one ${ }^{13)}$ does.
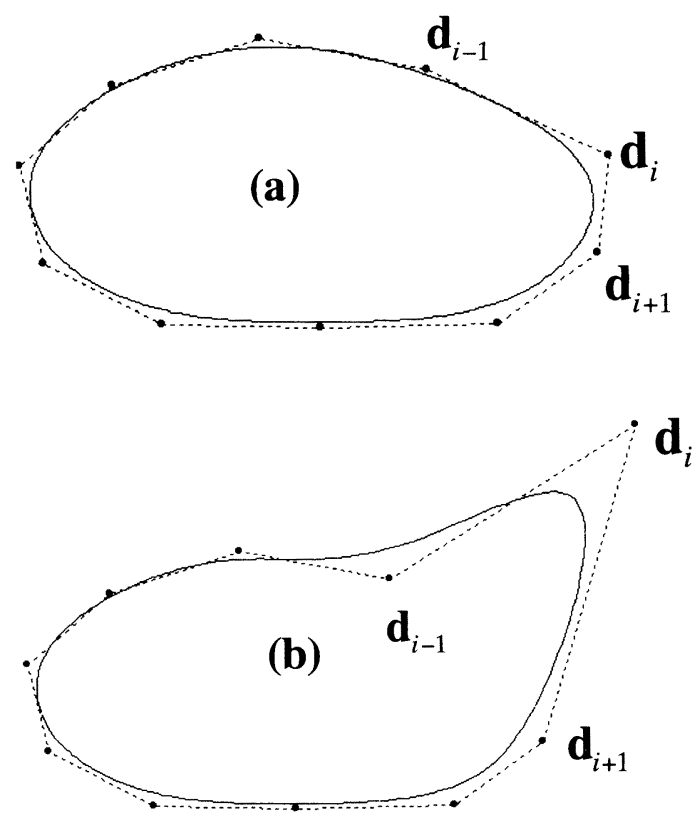

Fig. 1 The geometrical dependence of a B-spline curve on its coefficients; see formula ( 1 ) 


$$
\begin{aligned}
& B_{i, k}^{N}(u)=\sum_{j=1}^{N+1} b_{i, N}^{k, j} \cdot u^{N+1-j}, u \in\left[u_{i+k-1}, u_{i+k}\right), \\
& k=1 \cdots N+1 \\
& b_{i, N}^{k, p}=\Delta_{i}^{N}\left(b_{i, N-1}^{k, p}-b_{i, N-1}^{k, p-1} \cdot u_{i}\right) \\
& \quad-\Delta_{i+1}^{N}\left(b_{i+1, N-1}^{k-1, p}-b_{i+1, N-1}^{k-1, p-1} \cdot u_{i+N+1}\right) \\
& b_{i, 0}^{1,1}=1, \\
& b_{i, N-1}^{k, p}=0 \text { if } k<1 \text { or } p<1 \text { or } k>N \text { or } p>N(4) \\
& \Delta_{i}^{j}=\frac{1}{u_{i+j}-u_{i}}
\end{aligned}
$$

Figure 2 shows samples of four basis functions of different degrees.

The definition (1) is extended for the surface approximation by using the bi-cubic tensor product $\mathrm{B}^{-}$ spline surface

$$
\boldsymbol{r}(u, v)=\sum_{j=0}^{M+1 L+1} \sum_{i=0}^{2} \boldsymbol{d}_{i j} B_{i}^{3}(u) B_{j}^{3}(v)
$$

where $L$ and $M$ is the number of the data points to interpolate in $u$ and $v$ parametric directions respectively.

\section{B-spline surface fitting}

A single $\mathrm{B}^{-}$spline patch approximates each smooth part of the ship hull in the present method. The pictures in figure 3 show the result of so ${ }^{-}$called surface fitting for four types of hull forms (The ship hulls are depicted by two sets of parametric lines). The surface fitting represents a process, which determines the coefficients $\boldsymbol{d}_{i j}$ in formula ( 6 ) for any particular surface, given by external data. If $\boldsymbol{r}_{k l}=\left(x_{k l}, y_{k l}, z_{k l}\right)$ is a three-dimensional set of surface data points in the coordinate system shown in figure 6 , and $\left(u_{k} \times v_{i}\right)$ is an arbitrary uniform set of parameters, then solving the over-determined linear system of eqs. $(7)$ could do the fitting ${ }^{14)}$.

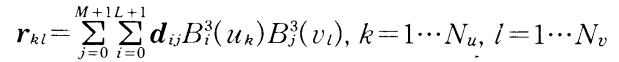

The only restriction is that the number of the data points must be larger or equal to the number of the $\mathrm{B}^{-}$ spline coefficients, or $N_{u} N_{v} \geq(M+2)(L+2)$.

For our needs we developed a "skinning" type method $^{15)}$ for the B-spline surface fitting. The method could be described as follows: At first, we approximate each waterline of the ship by a two-dimensional Bspline curve. A subroutine, based on the de Boor's

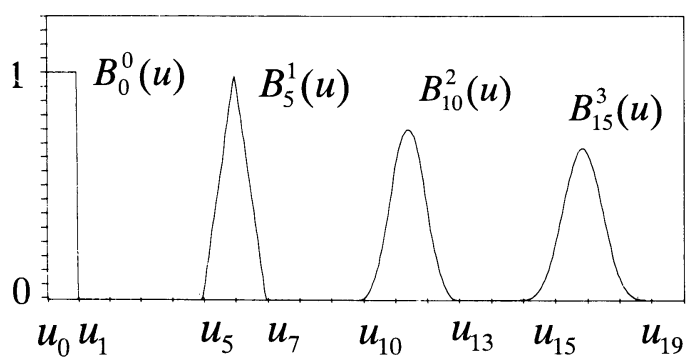

Fig. 2 Basis functions of various degrees; see formula (1) algorithm ${ }^{16)}$, performs this approximation. Then we extend the $\mathrm{B}^{-}$-spline to a tensor product by approximating in a vertical direction. In this case we have $N_{u}=M$, $N_{v}=L$ and the de Boor subroutine does not determine all of the coefficients. It returns the coefficients $\boldsymbol{d}_{i j}=$ $\left(X_{i j}, Y_{i j}, Z_{j}\right), i=1 \cdots M, j=1 \cdots L$, where $Z_{j}$ is the depth of $j^{\text {th }}$ waterline. The rest of the coefficients are obtained by using natural spline end-conditions, i.e. imposing zero second derivatives for the $\mathrm{B}$-spline on the surface boundaries. The "skinning" approach is effective, because De Boor's algorithm is more stable numerically than the direct solution of the system ( 7 ) is. In our case, it reduces the fluctuations of the spline over the flat segments of the ship.

The best approximation has been achieved by using the offset data of equally spaced waterlines in vertical direction. In this case, $z(v)$ becomes a linear function and $z\left(v_{j}\right)=Z_{j}$. Otherwise, we might have to interpolate new waterlines, with depths equal to $z\left(v_{j}\right)$, from the offset data. The approximation can be improved further manually by using the interactive visual technique, shown in figure $1(a)-(b)$. Such an approach has been implemented mainly for the corrections of the boundary line of the ship on the bow and the stern. Figure 3 shows the result of the fitting, applied to the following cases :

(a) Wigley model, Length $\mathrm{L}=100 \mathrm{~m}$, beam $\mathrm{B}=10$ $\mathrm{m}$, draft $\mathrm{d}=6.25 \mathrm{~m}, \mathrm{~L} / \mathrm{B}=10, \mathrm{~B} / \mathrm{d}=1.6, C_{B}=$ 0.444 , the form of the hull is described by $Y(x$, $z)=(B / 2)\left(1-(z / d)^{2}\right)\left(1-(2 x / L)^{2}\right)$, half of the hull is approximated by a single patch, $N_{u} \times N_{v}$

WIGLEY

(a)

(b)

SERIES 60

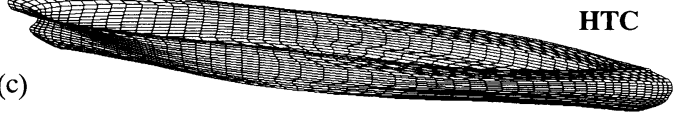

(d)

SR221B

Fig. 3 B-spline approximation of Wigley model ( a ), Series 60 (b), HTC (c) and SR221B-tanker (d) hulls 
$=21 \times 5$ data points, $20 \times 4=80$ panels (A panel in the parametric space is defined as $\left[u_{i}, u_{i+1}\right)$ $\left.\times\left[v_{i}, v_{i+1}\right)\right), 23 \times 7 \mathrm{~B}$-spline coefficients.

(b) Series $60, \mathrm{~L}=123.75 \mathrm{~m}, \mathrm{~B}=16.26 \mathrm{~m}, \mathrm{~d}=6.5 \mathrm{~m}$, $\mathrm{L} / \mathrm{B}=7.61, \mathrm{~B} / \mathrm{d}=2.5, C_{B}=0.600$, half of the hull is approximated by a single patch, $N_{u} \times N_{v}$ $=35 \times 7$ data points, $34 \times 6=204$ panels, $37 \times 9 \mathrm{~B}$ -spline coefficients.

( c) HTC (Hamburg Test Case) container ship, $\mathrm{L}=$ $153.7 \mathrm{~m}, \mathrm{~B}=27 \mathrm{~m}, \mathrm{~d}=10.55 \mathrm{~m}, \mathrm{~L} / \mathrm{B}=5.69, \mathrm{~B} /$ $\mathrm{d}=2.56, C_{B}=0.645$, half of the hull is approximated by a single patch, $N_{u} \times N_{v}=24 \times 13$ data points, $23 \times 12=276$ panels, $26 \times 15 \quad \mathrm{~B}$-spline coefficients.

(d) SR221B tanker, $\mathrm{L}=320 \mathrm{~m}, \mathrm{~B}=58 \mathrm{~m}, \mathrm{~d}=19.30$ $\mathrm{m}, \mathrm{L} / \mathrm{B}=5.52, \mathrm{~B} / \mathrm{d}=3.00, C_{B}=0.802$, half of the hull is approximated by a single patch, $N_{u} \times N_{v}$ $=24 \times 8$ data points, $23 \times 7=161$ panels, $26 \times 10$ B-spline coefficients.

One can see that a much smaller number of panels has
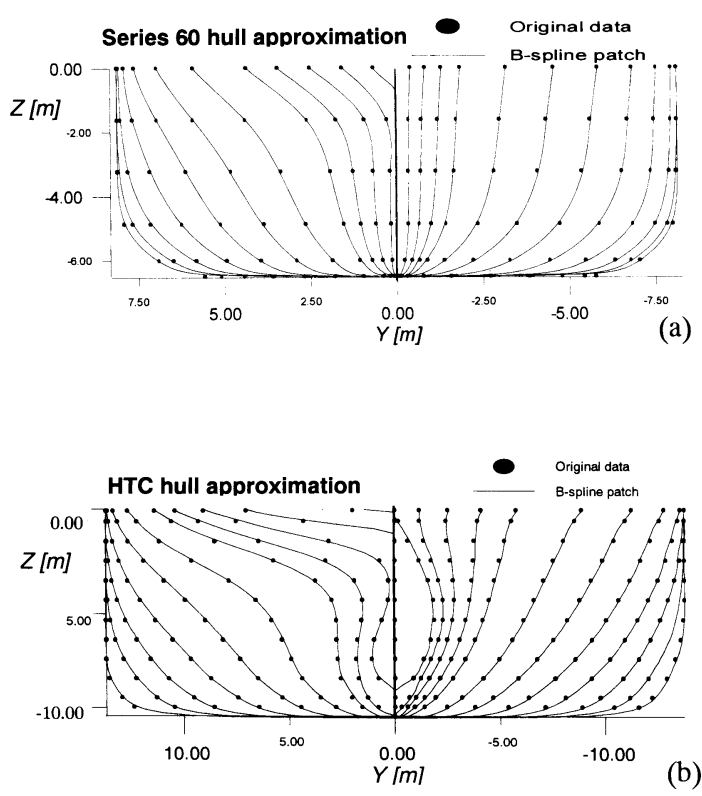

Fig. 4 B-spline approximation of Series 60 (a) and HTC ( b ) hulls by a single patch

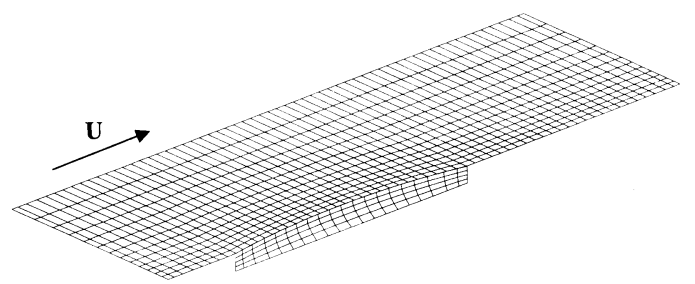

Fig. $5 \mathrm{~B}^{-}$spline approximation of the free-surface domain $(60 \times 13$ panels $)$ been used than in lower-order panel methods.

In order to keep the simplicity of this presentation, we restrict our work to the ships with entirely smooth hulls. Their geometry is approximated by a single bi cubic $\mathrm{B}^{-}$spline patch. Thus, we have $C^{2}$-continuity of the hull surface or continuously distributed normal vector and curvature over the whole ship hull, except for its boundary line. The accuracy of the approximation is sufficient for computing correct wave resistance, as one can see from the calculated results in section 8 . We compare the original ship sections with those obtained by a single patch approximation in figure 4 . It should be noted that we could approximate not only entirely smooth surfaces by a single patch, but some surfaces with discontinuous curvature as well. The use of repeated parametric knots allows a jump of the normal vector on a prescribed curve of the hull surface $^{13)}$.

In order to complete the geometry of the problem, a half of the free-surface domain must be approximated as well ; see figure 5. The two B-spline tensor products, which describe the geometry of our problem, are

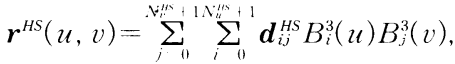

$$
\begin{aligned}
& u \in\left[u_{1}, u_{N H^{s}}\right], v \in\left[v_{1}, v_{N H^{s}}\right]
\end{aligned}
$$

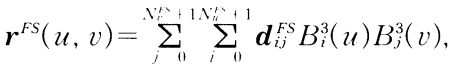

$$
\begin{aligned}
& u \in\left[u_{1}, u_{N \ell t} s\right], v \in\left[v_{1}, v_{N f: s}\right]
\end{aligned}
$$

where the superscript HS denotes the hull-surface and FS denotes the free-surface.

\section{Approximation of the potential}

This paper is directed to solving the ship wavemaking problem. To do so, we will solve inviscid flow surrounding the ship. It can be reduced to the problem of finding the velocity potential. In our case, the potential is a scalar function, distributed over the two surfaces described by expressions ( 8$)-(9)$. We will approximate it by the following tensor product parametric $\mathrm{B}$-splines

$$
\begin{aligned}
& \varphi^{H S}(u, v)=\sum_{j}^{N_{0}^{\prime \prime \prime}} \sum_{i}^{1} \sum_{0}^{m, n} \Phi_{i j}^{H S} B_{i}^{3}(u) B_{j}^{3}(v), \\
& u \in\left[u_{1}, u_{N / t s}\right], \quad v \in\left[v_{1}, v_{N H / s}\right] \\
& \varphi^{F S}(u, v)=\sum_{j} \sum_{i}^{1} \sum_{i=1}^{1} \Phi_{i j}^{F S} B_{i}^{3}(u) B_{j}^{3}(v) \text {. } \\
& u \in\left[u_{1}, u_{N F s}\right], \quad v \in\left[v_{1}, v_{N F} s\right]
\end{aligned}
$$

where the coefficients $\Phi_{i j}^{H S}, \Phi_{i j}^{F S}$ are the unknown characteristics of the flow. The next sections will show how these can be found. When comparing eq. (10) to eq. ( 8 ) and eq. (11) to eq. (9), one can see that the same parametric space connects the potential with geometry. Considering the $\mathrm{B}^{-}$spline properties, the above approximation ensures continuous distribution of the fluid velocity $\boldsymbol{v}=\operatorname{grad} \varphi$ over the two boundary surfaces and in the enclosed volume as well. 


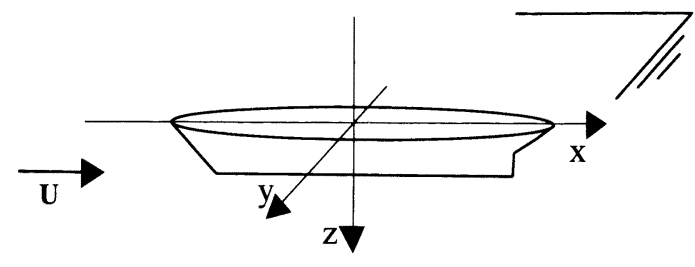

Fig. 6 The Cartesian coordinate system

\section{Formulation of boundary-value problem}

The fluid surrounding the ship is assumed to be incompressible and inviscid. The flow is irrotational, steady, and it has a constant speed $\boldsymbol{U}=(U, 0,0), U>0$ at infinity ; see figure 6 . Then the governing equation for our mathematical model is the Laplace equation:

$$
\Delta \phi(\boldsymbol{r})=0, \quad \boldsymbol{r}=(x, y, z)
$$

According to the Neumann-Kelvin formulation ${ }^{17)}$, the potential $\phi$ can be decomposed into a potential of the basic flow $U x$ and a potential $\varphi$ due to the fluid disturbance caused by the ship :

$$
\phi=U x+\varphi
$$

When assuming $|\nabla \varphi| \ll|\nabla \phi|$ the nonlinear free-surface boundary condition is linearized as

$$
U^{2} \varphi_{x x}+g \varphi_{z}=0, \quad z=0
$$

In order to enforce a radiation condition we will impose the additional conditions

$$
\varphi_{x}=0 \quad \varphi_{x x}=0
$$

as end conditions of the spline (11) on the upstream end of the free-surface ${ }^{7}$. The restrictions in eq. (15) have the meaning of zero wave amplitude and zero strength of the free-surface sources, according to the adopted Neumann-Kelvin formulation of the problem. Geometrically, it means that the free-surface turns into a horizontal plane in upstream direction. Since the wave amplitude is a continuous function, the waves do not spread forward.

So far we have discussed only the boundary conditions on the free-surface described by formula (9). The presence of a ship in the flow is represented by the rigid-body condition

$$
\frac{\partial \varphi}{\partial n}=\boldsymbol{U} \cdot \boldsymbol{n}
$$

where $\boldsymbol{n}=\left(n_{1}, n_{2}, n_{3}\right)=\left(\boldsymbol{r}_{u}^{H S} \times \boldsymbol{r}_{v}^{H S}\right) /\left|\boldsymbol{r}_{u}^{H S} \times \boldsymbol{r}_{v}^{H S}\right|$ is an unit normal vector to the ship hull surface described by eq. (8). The vector $\boldsymbol{n}$ is directed into the fluid domain.

The above boundary conditions allow transforming the Laplace equation into the following integral equation

$$
\begin{aligned}
& 2 \pi \varphi\left(\boldsymbol{r}_{c}\right)+\frac{U^{2}}{g} \int_{S^{F S}} \frac{\varphi_{x x}(\boldsymbol{r})}{\left|\boldsymbol{r}-\boldsymbol{r}_{c}\right|} d S-\int_{S^{H} \cup_{\cup S^{F S}}} \varphi(\boldsymbol{r}) \frac{\left(\boldsymbol{r}-\boldsymbol{r}_{c}\right) \cdot \boldsymbol{n}}{\left|\boldsymbol{r}-\boldsymbol{r}_{c}\right|^{3}} d S \\
& -\boldsymbol{U} \cdot \int_{S^{H S}} \frac{\boldsymbol{n}}{\left|\boldsymbol{r}-\boldsymbol{r}_{c}\right|} d S=0
\end{aligned}
$$

where the subscript $c$ will distinguish a collocation point. The second $x$-derivative of the potential is written by

$$
\varphi_{x x}=A \varphi_{u u}+B \varphi_{u v}+C \varphi_{v v}+D \varphi_{u}+E \varphi_{v}
$$

where $A, B, C, D$ and $E$ depend on the local freesurface geometry. Note that we encounter the full expression (18) rarely on the grid. In the areas where the gridlines are parallel to the $x$-axis, it can be proved that $B=C=E=0$ and those should not be calculated.

After applying the collocation method ${ }^{18)}$ for the integral eq. (17), we obtain the linear system of eqs. (19) for the unknown B-spline coefficients $\Phi_{i j}^{H S}, \Phi_{i j}^{F S}$.

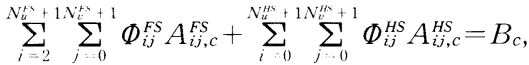

$$
\begin{aligned}
& c=1 \cdots N_{c} \\
& \Phi_{0 j}^{F S}=\Phi_{1 j}^{F S}=\Phi_{2 j}^{F S}, \quad j=0 \cdots N_{v}^{F S}+1 \\
& A_{2 j, c}^{F S}=A_{0 j, c}^{F S}+A_{1 j, c}^{F S}+A_{2 j, c}^{F S}, \quad j=0 \cdots N_{v}^{F S}+1 \text {, } \\
& c=1 \cdots N_{c}
\end{aligned}
$$

$N_{c}$ is the number of collocation points. The influence coefficients $A_{i j, c}^{F S}, A_{i j, c}^{H S}$ and $B_{c}$ are determined by substituting formulas (10)-(11) in eq. (17). Setting the first three unknowns on the free-surface to be equal enforces the radiation condition (15) if the spline (11) is chosen to be uniform.

All of the integration over the ship hull surface and some of the free-surface integrals in eq. (17) are too complex to be evaluated analytically. Besides, the unknown $\mathrm{B}^{-}$-spline coefficients have grater number than the number of panels, so that one collocation point per panel is insufficient to make the system (19) determined. In order to get more equations we use either more collocation points or additional spline end conditions. That is why the three most important points for the numerical stability and efficiency of the present method are :

(a) Accuracy and efficiency of the numerical integration,

(b) Number and position of the collocation points,

(c) Number and type of the additional spline end conditions

Section 6 shows how we perform the integration of the Rankine source coefficients. The number and position of the collocation points $\boldsymbol{x}_{c}$ as well as the additional spline end conditions will be discussed in section 7 .

The system (19) is solved by the least-squares method. After solving the system, the hydrodynamic characteristics are to be evaluated. We give the expressions (20) for the wave elevation $\zeta$, pressure $p$ and wave resistance $R$, which relate to the Neumann-Kelvin formulation. These functions will be used to compare the present model to the experimental data as well.

$$
\begin{aligned}
& \zeta=-\frac{U}{g} \varphi_{x}=-\frac{U}{g} \frac{\varphi_{u}^{F S} y_{v}^{F S}-\varphi_{v}^{F S} y_{u}^{F S}}{x_{u}^{F S} y_{v}^{F S}-x_{v}^{F S} y_{u}^{F S}} \\
& p(x, y, z)=-\rho\left(U \varphi_{x}-\frac{1}{2}(\nabla \varphi)^{2}\right), C_{P}=\frac{p}{\frac{1}{2} \rho U^{2}}, \\
& R=-\iint_{S^{H S}} p n_{1} J^{H S} d u d v, \quad C_{W}=\frac{R}{\frac{1}{2} \rho U^{2} S^{H S}},
\end{aligned}
$$

In the above expression $S^{H S}$ is the surface area of the 
ship, $g$ is the gravitational acceleration, $\rho$ is the fluid density and $J^{H S}=\left|\boldsymbol{r}_{u} \times \boldsymbol{r}_{v}\right|$ is the surface area element. Although the nonlinear term $(\nabla \varphi)^{2}$ in the pressure definition does not relate to the adopted lineariztion, it improves the result especially for the practical hull forms. We will discuss the effect of this term in section 8. The integral in eq. (20) is evaluated by Kronrod quadrature. The gradient of the potential is obtained from the following system of equations

$$
\begin{aligned}
& \nabla \varphi \cdot \boldsymbol{r}_{u}=\varphi_{u}, \\
& \nabla \varphi \cdot \boldsymbol{r}_{v}=\varphi_{v}, \\
& \nabla \varphi \cdot\left(\boldsymbol{r}_{u} \times \boldsymbol{r}_{v}\right)=0
\end{aligned}
$$

\section{Integration of singularities}

The integration of the Rankine source coefficients is performed over each panel separately. The numerical procedures are divided into three types according to the behavior of the integrated function:

(a) Far-field integration; where the function is regular,

(b) Near-field integration; where the function is near-singular,

(c) Singular integration; when the collocation point is on the integrated panel.

In the integration ( $\mathrm{a}$ ), the collocation point is relatively far from the integrated panel and $3 \times 3$ points Gauss-Legendre quadrature is used in this case. The values of $\boldsymbol{r}^{H S}, \boldsymbol{r}^{F S}, \boldsymbol{n}, J^{H S}$ and $J^{F S}$, evaluated in the Gaussian points of each panel, should be stored in advance ${ }^{16,10)}$. Otherwise, they would be evaluated repeatedly in the calculations.

In case ( $b$ ), the collocation point is relatively close to the integrated panel and the behavior of the function is too singular to be treated as in case (a). This problem could be resolved by adaptive division of the integrated area ${ }^{9,10,19)}$. The resulting smaller areas satisfy the far-field definition.

In order to evaluate the singular integral in case (c) accurately, the collocation point is isolated in a small area. This area could be integrated using the numerical integration developed by Cerrolaza ${ }^{20)}$. Another way to integrate the area is to remove the singularity analytically ${ }^{10-11)}$, and then the resulting non-singular integrals could be evaluated by Gauss-Legendre quadrature. We tested both techniques and adopted the second one, which is faster, in our opinion.

\section{Collocation points and spline end conditions}

Panel methods usually place one collocation point on each panel. In our case, however, the number of panels is $\left(N_{u}^{H S}-1\right)\left(N_{v}^{H S}-1\right)+\left(N_{u}^{F S}-1\right)\left(N_{v}^{F S}-1\right)$ and the number of the unknown B-spline coefficients is $\left(N_{u}^{H S}+N\right.$ $-1)\left(N_{v}^{H S}+N-1\right)+\left(N_{u}^{F S}+N-1\right)\left(N_{v}^{F S}+N-1\right)$, where $N$ is the degree of approximation. That is why the use of one collocation point per panel makes the system of equations underdetermined for $N>0$. One way to resolve this problem is to force additional end condi- tions to the $\mathrm{B}^{-}$spline functions. For example, Sclavounos and Nakos ${ }^{7)}$ imposed the following freesurface end conditions for $N=2: \varphi_{x}^{F S}=0$ and $\varphi_{x x}^{F S}=0$ on the upstream end of the free-surface; $\varphi_{y y}^{F S}=0$ on the transverse end of the free-surface grid; $\varphi_{y}^{F S}=0$ on the centerline upstream and downstream of the hull ; natural end conditions on the bow, stern and bottom lines of the hull; $\varphi^{F S}=\varphi^{H S}$ and $\varphi_{n}^{F S}=\varphi_{n}^{H S}$ on the waterline (where $n$ represents the normal vector to the hull surface). They use a large number of flat panels for the geometry approximation, whereas we have two Bspline patches. Therefore we cannot align the hull surface panels with free- ${ }^{-}$surface panels on the waterline as they do. We impose so-called "not-a-knot" conditions $^{13)}$ instead of their last two conditions, and instead of the natural conditions, too. Knots are points in the parametric space where the highest derivatives of the spline function are discontinuous. "Not-a-knot" conditions force the polynomial segments (the end segments in our case) to merge into one piece by setting the highest derivatives to be equal, i.e. each of the knots involved is "not-a-knot" anymore. These conditions are more neutral physically than the natural ones are. The natural conditions impose zero second derivatives on the end lines. It means, we have extremal values of the first derivatives there, or we have extremal veloc-
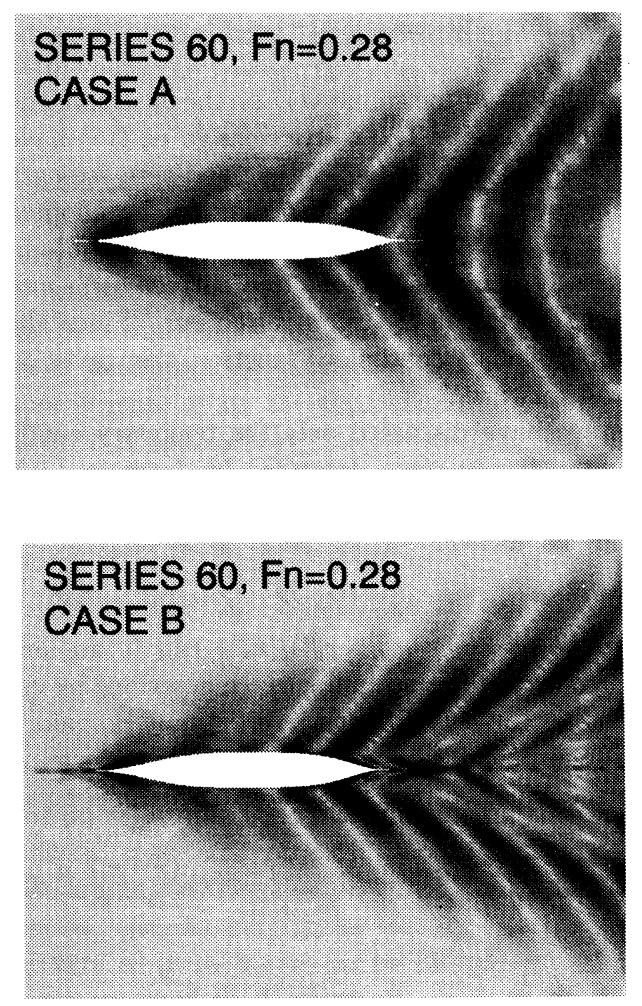

Fig. 7 Series 60 wave patterns, $F n=0.28$ : ( a ) case A $\left(C_{W}=1.19 \cdot 10^{-3}\right)$; ( b ) case B $\left(C_{W}=0.75 \cdot 10^{-3}\right)$ 
ities and pressure on these lines, which is not natural restriction. "Not-a-knot" conditions do not influence the physical sense of the solution. They make the spline less flexible, but they preserve the behavior of the first derivatives. The number of the" not-a-knot" conditions is not restricted as well, i.e. they can generate more additional equations and could allow approximation with degrees $N>2$.

Another approach is the use of more than one collocation point per panel ${ }^{10)}$. If we use this approach, then we do not need the additional spline end conditions, except for the radiation ones. A stable scheme is achieved by putting four or nine control points on each panel. All of the points are situated at the zeroes of the Legendre polynomials. Due to the larger number of linear equations, this algorithm takes about 3 times more CPU time to build and solve the system of equations (19). The larger number of linear equations also leads to a weaker radiation condition. However, the algorithm generally converges better and is less grid-dependent. In figure 7, "case A" shows a wave pattern for Series 60 $(F n=0.28)$, calculated by a 1 -point-per-panel bi-quadratic scheme with additional spline end conditions, whereas "case B" represents a 4-point per-panel bicubic scheme, without additional spline end conditions. One can see that the bi-cubic approximation can simulate better the diverging wave system, but slightly violates the radiation condition upstream. Probably it causes the resistance coefficient to be lower with about $35 \%$, than in "case A". All the calculations in section 8 are carried out by the "case A" scheme. We still use "case B" to verify the results for it has different sources
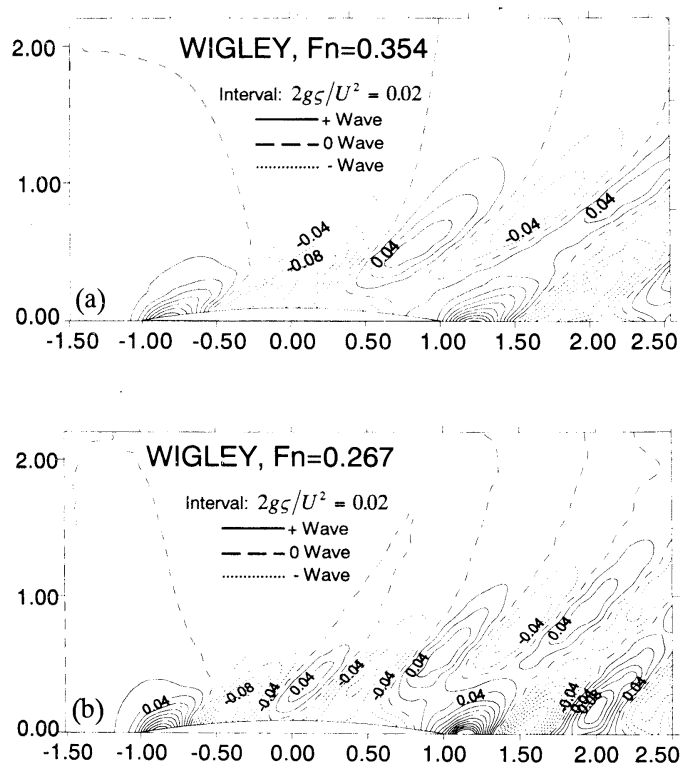

Fig. 8 Wigley model wave patterns: (a) $F n=0.354$; (b) $F n=0.267$ of numerical error.

\section{Numerical results}

This section starts with the calculated results for the Wigley model. Wave patterns for two different Froude numbers are shown in figure 8 . The illustrated dimensions of the free-surface domain reduce the wave reflections due to its truncation in transverse direction $^{21}$. There are no waves upstream in these examples. Following Nakos, we generate the freesurface panels to be evenly distributed in longitudinal direction. Each panel becomes by $10 \%$ wider in transverse direction. The characteristics of the grid are :

$$
\alpha=\frac{\Delta x}{\Delta y}=1, \quad F_{h}=\frac{U}{\sqrt{g \Delta x}}=0.67 \sim 1.56
$$

where $\Delta x$ and $\Delta y$ represent the dimensions of the freesurface panels on the waterline ${ }^{22)}$. Figure 5 illustrates an example of a mesh of this type. Similar panel
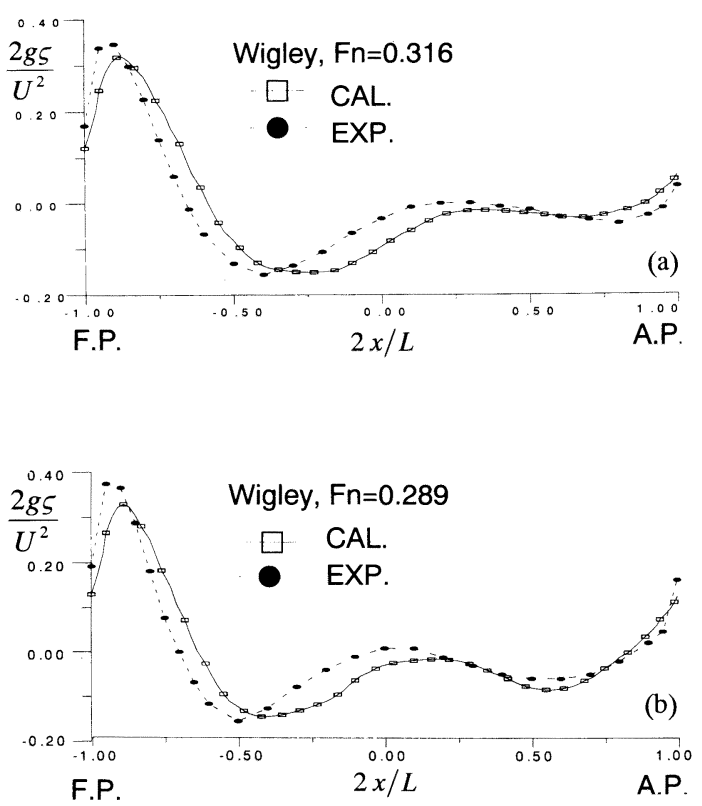

Fig. 9 Wigley model wave elevation along the waterline: ( a ) $F n=0.316$; (b ) $F n=0.289$

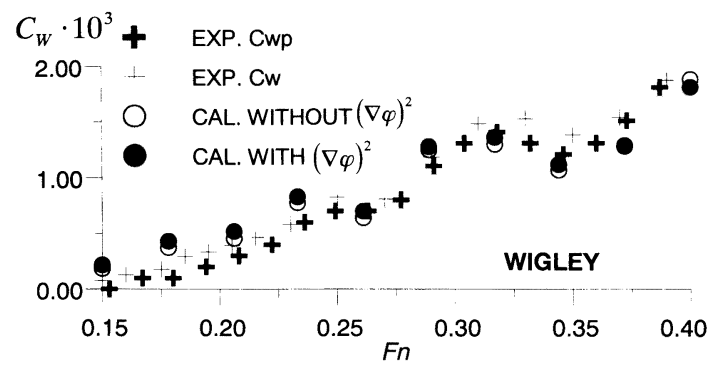

Fig. 10 Wave resistance of Wigley model 
arrangements will be used for all calculations in this section. In the case of the Wigley model, $40 \times 13=520$ panels are placed on the area $(y>0, z=0)$. Such a number ensures convergence for slender ships. Figure 9 shows agreement of the wave amplitudes, measured on the hull surface, with the experimental data ${ }^{23)}$. We compare the wave resistance with and without using the quadratic term in figure 10 ; see formula (20). Both results are close to the experiment. Here, as one can see, the term does not make any significant difference, but it will play a more important role for the practical hull forms.

The solution has been approximated by uniform spline so far. It is possible for the Wigley model because of its geometric simplicity. For the realistic ship forms we approximate the potential on the hull surface by non-uniform splines with higher density of the grid at the bow and stern. Now, the panels of the geometry and potential have different number and geometry ${ }^{12)}$. Besides, the panels of the free-surface potential and hull potential should not be necessarily aligned on the waterline in our case. Thus, we avoid the magnification of some artificial short scale waves by generating a coarser grid for the free-surface. That does not affect the grid on the hull, and we do not lose convergence.

Some local instabilities of the solution, arising on the waterline near the bow and stern, can spread downstream. This happens when the density of the freesurface mesh is selected higher than needed. This also happens for some specific Froude numbers, which produce a lower stern wave, or when modifying the form of the stern. In order to prevent that, we use relatively wider (in transverse direction) free-surface panels near the stern. In other words, the aspect ratio $\alpha$ is reduced, while $F_{h}$ remains unchanged in this area. It gives satisfactory results in all cases described in section 8 . Hong, S. and Choi, $\mathrm{H}^{8}{ }^{8}$, take special care of the stern waves as well. We should note that the "Case B" algorithm is more stable numerically in this case, because the mentioned error remains local.

The solution for Series 60 is calculated by using (FS panels $)+(H S$ panels $)=40 \times 10+34 \times 6=604$ panels for potential approximation. It can be compared with the greater number of $150 \times 20+75 \times 10=3750$ panels used by a lower-order panel method ${ }^{24)}$ for solving the same

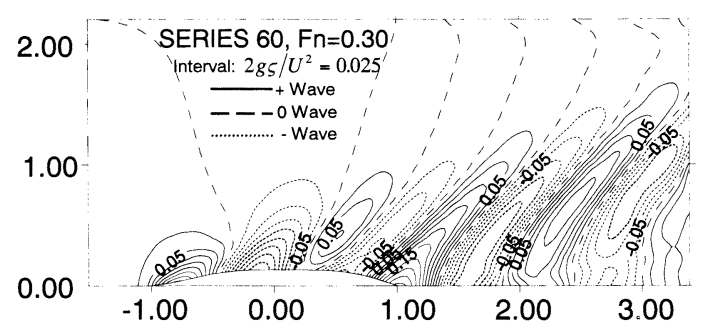

Fig. 11 Series 60 wave pattern for $F n=0.30$ problem. The higher-order geometry maintains the accuracy when using as many as 6 times fewer panels, in our case. The numerical results, shown in figures 11 $\sim 13$, are related to Series 60 . The wave pattern in figure 11 illustrates the implementation of the radiation condition. There is some wave reflection from the transverse end of the grid. In our opinion, however, the reflection does not visibly affect the evaluation of the wave resistance. The graphs in figure 12 show an agreement with the experimental data of the calculated wave elevation along the waterline. The calculated wave resistance is in agreement with the experiment too, as it is shown in figure 13. The nonlinear term in the pressure integration is not of big importance.

Figures 14 16 illustrate calculated results for HTC container ship. These results are compared with the experiment $^{25)}$ and with a lower-order panel method $(\mathrm{LOPM})^{26)}$. The number of panels in figure 14 is $70 \times$
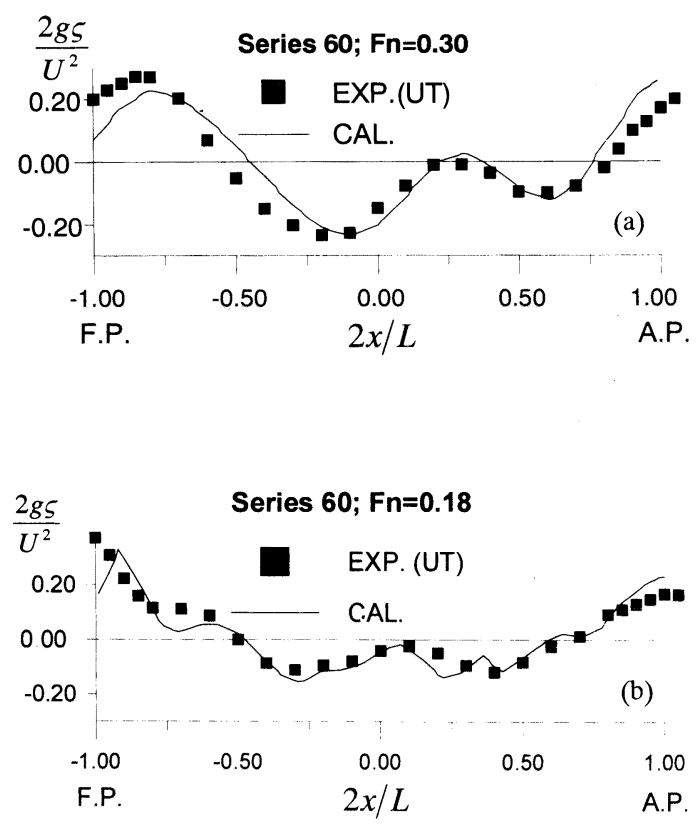

Fig. 12 Series 60 wave elevation along the waterline: (a) $F n=0.30$; (b) $F n=0.18$

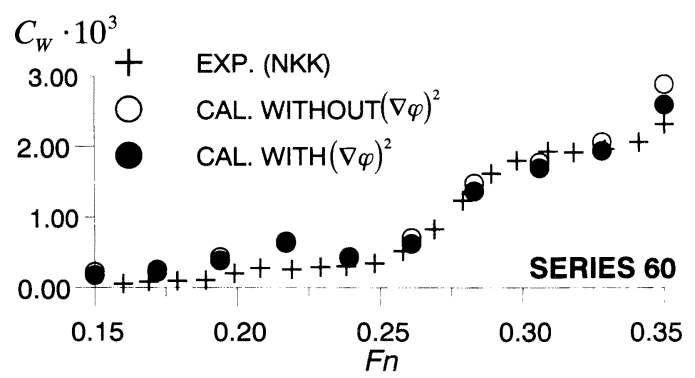

Fig. 13 Wave resistance of Series 60 


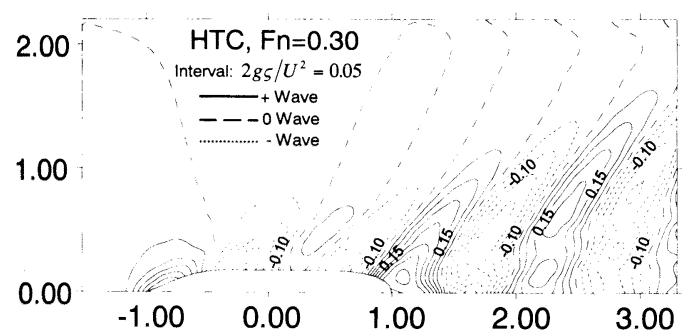

Fig. $14 H T C$ wave pattern for $F n=0.30$

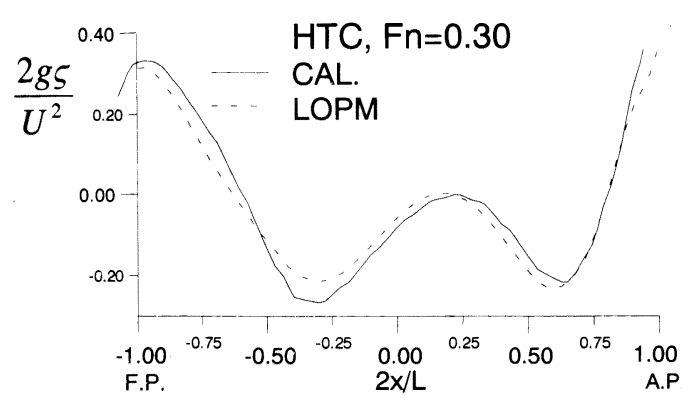

Fig. $15 H T C$ wave elevation along the waterline for $F n=0.30$

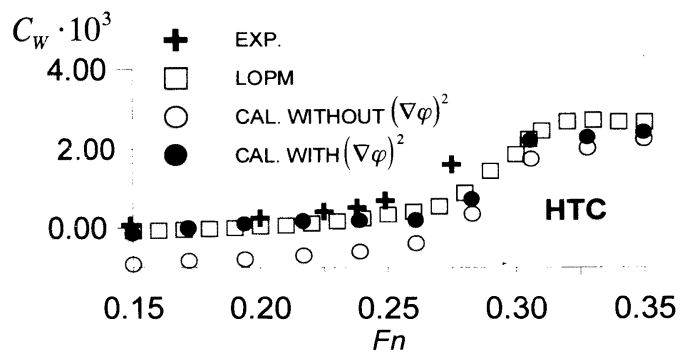

Fig. 16 Wave resistance of HTC container ship

$14+50 \times 15=1730$. Figure 15 shows that wave amplitudes calculated by the present method are larger, in comparison with the LOPM. The wave resistance coefficient in figure 16 is evaluated by using the formula (20) with and without the nonlinear term $(\nabla \varphi)^{2}$. Since the normal vector changes rapidly in a vertical direction above the bulb, a more significant $\varphi_{z}$ component is evaluated there. The component becomes relatively higher for the lower Froude numbers, where the value of $\varphi_{x}$ decreases. Thus, higher pressure is calculated in this area when using the term. We should also note that dropping the nonlinear term produces greater errors in our method than in the case of a double-body model panel method. A better result is evaluated by extending the upstream free-surface area to $0.5 \mathrm{~L}$. The upstream extension is important especially for the lower Froude numbers, because the forward wave spreads wider in

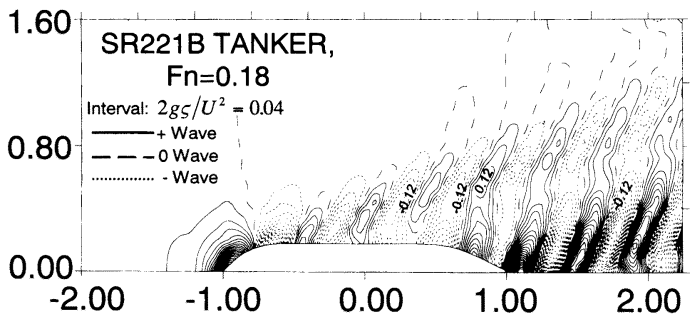

Fig. $17 S R 221 B$ wave pattern for $F n=0.18$
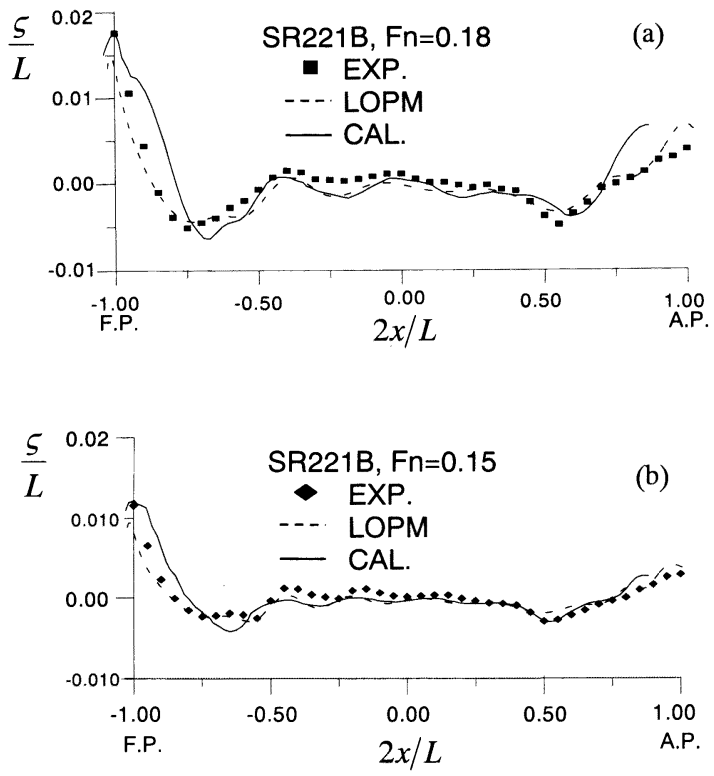

Fig. $18 S R 221 B$ wave elevation along the waterline: (a) $F n=0.18$; (b) $F n=0.15$

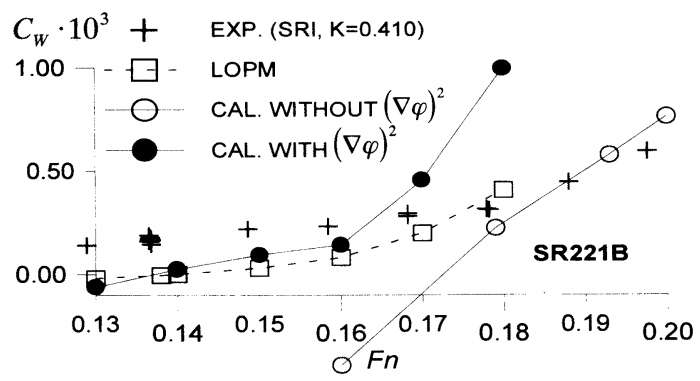

Fig. 19 Wave resistance of SR221B tanker

this case and the radiation condition would affect it.

To solve the flow surrounding SR $221 \mathrm{~B}$ tanker, we increase the HS panels of the potential to $52 \times 10=520$. That gives a better resolution of the solution on the waterline. In this case, these panels are much more than the panels of HS geometry : $23 \times 7=161$. The panels of potential also have higher density on the cylindrical 
part of the ship than the panels of the geometry. We place $90 \times 15=1350$ panels on the free-surface to catch the shorter wave lengths. Figure 17 illustrates that the higher-order approximation allows well-shaped transverse and diverging waves, using $2 \sim 3$ times smaller number of panels than LOPM usually needs. The bow waves in figures 18 ( $a) \sim(b)$ are again higher than the waves calculated by the lower-order panel method, but the agreement with the experiment ${ }^{27)}$ has been less so far. The stern wave is shifted forward, probably due to the mentioned numerical error in the pressure on the waterline. When using the quadratic term the resistance coefficient is positive almost down to the limit where convergence fails (about $F n=0.12$ ) ; see figure 19. However, it increases rapidly for $F n=0.18$. The peak is probably due to the limit of the adopted Kelvin's free-surface condition. A similar result is reported by Raven $^{28)}$ for a tanker. In our case, the calculated wave resistance is negative for $F n<0.17$ when not using the nonlinear term, but it shows a stable trend, close to the experiment for $F n \geq 0.18$. One can see that the nonlinear term slightly decreases the wave resistance for slender ships (Wigley and Series 60), whereas it increases the resistance of the practical hull forms with bulbs (HTC and SR $221 \mathrm{~B}$ ).

The $\mathrm{B}^{-}$-spline approximation of the surfaces depends on the topology of the described object. The numerical results relating to four realistic ship hulls, which have identical topologies, are described in this section. None of those, however, could be solved analytically. Therefore, we have difficulties doing a systematic analysis, concerning the efficiency of the present method for a prescribed accuracy in this stage. Since we use a different compiler for the code generation, we could not compare the efficiency of the method with the other panel methods, either. What we could note is that the CPU time should be divided into two parts: Part 1, which represents the generation of the matrixes $\left\{A_{i j, c}\right\}$ and $\left\{B_{c}\right\}$, and Part 2, which goes for solving the linear system ; see eq. (19). The CPU time of the Part 1 takes about $85 \%$ of the full process. For example, in the illustrated case of Series 60 , the first part takes about 9 min. The code here is generated by a Borland Delphi (Visual Pascal) compiler. A Fortran based DLL performs the second part in $1.5 \mathrm{~min}$. That means, we need 1.5 min to calculate every additional Froude number. This estimate is valid for a CPU : Pentium-III-MMX, $750 \mathrm{MHz}$. A more detailed discussion about the efficiency of solving two-dimensional flow (a hydrofoil) can be found in Hsin et $\mathrm{al}^{9)}$. A discussion about the efficiency of solving three-dimensional mathematical objects such as spheres, cubes, and cylinders by B-spline based Kelvin source panel method can be found in Maniar ${ }^{10)}$

\section{Conclusion}

The following conclusions can be obtained from this paper.

1. A new practical higher-order Rankine source method, fully based on $\mathrm{B}^{-}$-splines is presented in the paper.

2. A skinning type technique for $\mathrm{B}^{-}$-spline surface fitting of ship hulls is developed.

3. The present method produces better results for the two practical hulls (HTC and SR 221 B) if the nonlinear term of the solution stays in the pressure integration. Thus, the negative wave resistance evaluation is practically avoided. The influence of this term for the two slender hulls (Wigley and Series 60) is negligible.

4. The numerical results in section 8 prove that the global single patch approximation of four different hull surfaces works well in conjunction with the higher-order panel method. Thus, this method can be used for a smooth optimization of the ship hull design in the future.

\section{Acknowledgment}

The authors are grateful to the Ministry of Education, Culture and Science of Japan (Monbusho), whose doctoral course scholarship made the present research possible. The first author would like to thank also to Prof. M. Ikehata and Prof. H. Kai (Yokohama National University, Japan), Dr. S. Kjulevcheliev and Dr. Y. Yovev (Bulgarian Ship Hydrodynamics Center) for their advice and support.

\section{References}

1) Dawson, C. W., "A Practical Computer Method for Solving Ship-Wave Problems", Second International Conference on Numerical Ship Hydrodynamics, Berkeley, 1977.

2) Jensen, G., Mi, Z. - X. and Soding, H. "Rankine Source Methods for Numerical Solution of the Steady Wave Resistance Problem“, 16th Symposium on Naval Hydrodynamics, Berkeley, 1986

3) Jensen, P. S., "On the Numerical Radiation Condition in the Steady-State Ship Wave Problem", Journal of Ship Research Vol. 31, No. 1, 1987

4) Ando, J. and Nakatake, K., "A Method to Calculate Wave Flow by Rankine Source" (in Japanese), Transaction of the West-Japan Society of Naval Architects, No. 75, pp. 1-12, 1988.

5) Raven, H.C., "Variation on a Theme By Dawson", 17th Symposium on Naval Hydrodynamics, The Hague, The Netherlands, 1988.

6) Seto, H., "Some Considerations on the Basis of Rankine Source Methods and the Treatment of Open Boundaries in Steady Ship Wave Problems“" (in Japanese), Transaction of the West-Japan Society of Naval Architects, No.81, pp. 11-28, 1991.

7) Sclavounos, P. D., and Nakos, D. E., "Stability Analysis of Panel Methods for Free-Surface Flows with Forward Speed", 17th Symposium on Naval Hydrodynamics, The Hague, The Netherlands, 1988.

8) Hong, S., Choi, H., "Steady and Unsteady Ship 
Waves by a Higher-Order Boundary Element Method“, 20th Symposium on Naval Hydrodynamics, Santa Barbara, California, 1994.

9) Hsin, C. - Y., Kerwin, J. E. and Newman, J. N., “A Higher-Order Panel Method Based on Bsplines", Sixth International Conference on Numerical Ship Hydrodynamics, Iowa City, Iowa, 2 August 1993.

10) Maniar, H., “A Three Dimensional Higher Order Panel Method Based on B-splines", Ph. D. Thesis, Department of Ocean Engineering MIT, Cambridge, Massachusetts, USA. 1995.

11) Lee, C. - H., "Wave Interactions with Huge Floating Structures", Proceedings of 8th International Conference on the Behavior of Off-shore Structures. Delft, The Netherlands, 1997.

12) Lee, C. -H., Farina, L. and Newman, J. N., “A Geometry Independent Higher Order Panel Method and its Applications to Wave-Body Interactions“, 3rd Biennial Engineering Mathematics and Applications Conference, South Australia, 1998.

13) Farin, G., Curves and Surfaces for Computer Aided Geometric Design. A Practical Guide, Academic Press, Inc., 1990.

14) Cheng-Hung Huang, Cheng-Chia Chiang, and Shean-Kwang Chou "An Inverse Geometry Design Problem in Optimizing Hull Surfaces", Journal of Ship Research Vol. 42, No. 2, pp. 79-85, June 1998.

15) Nowacki, H., Oleksiewich, B., Bloor, M. I. G., Dekanski, C. W., Wilson, M. J., Computational Geometry for Ships, World Scientific Publishing Co. Pte. Ltd., 1995.

16) De Boor, C., A Practical Guide to Splines, Applied Mathematical Sciences, 27, SpringerVerlag, 1978.

17) Newman, J. N. Marine Hydrodynamics, The MIT Press, MIT, Cambridge, Massachusetts, 1977.

18) Brebbia, C. A. and Dominez, J., Boundary Elements An Introductory Course, Computational
Mechanics Publications, Southampton, 1989.

19) Press, W. H., Teukolsky, S. A., Vettering, W. T., Flannery, B. P., Numerical Recipes in FOR TRAN. The Art of Scientific Computing, Cambridge University Press, 1994.

20) Cerreloza, M. and Alarcon, E. "A Bi-cubic Transformation for the Numerical Evaluation of the Cauchy Principal Value Integrals in Boundary Elements“, International Journal for Numerical Methods in Engineering, Vol.28, pp. 987-999, 1989.

21) Nakos, D.E., and Sclavounos, P. D., "Ship Motions by a Three-dimensional Rankine Panel Method", 18th Symposium on Naval Hydrodynamics, USA, 1990.

22) Nakos, D. E., and Sclavounos, P. D., "On Steady and Unsteady Wave Patterns", J. Fluid Mech., Vol. 215, pp. 263-288, 1990.

23) Cooperative Experiments on Wigley Parabolic Models in Japan, prepared for the 17th ITTC Resistance Committee by Ishikawajima-Harima Heavy Industries Co., Ltd., Ship Research Institute, University of Tokyo and Yokohama National University, December 1983.

24) Nakos D. E., and Sclavounos, P. D., "Kelvin Wakes and Wave Resistance of Cruiser-and Transom-Stern Ships“, Journal of Ship Research Vol. 38, No. 1, pp. 9-29, 1994.

25) Bertram, V., Chao K. -Y., Lammers, G. and Laudan, J., "Experimental Validation Data of Free-Surface Flows for Cargo Vessels", Proceedings of CFD Workshop Tokyo 1994, Vol. 1

26) Suzuki, K., Iokamori N., "Studies on Minimization of Wave Making Resistance Based on Rankine Source Method“, Journal of the Society of Naval Architects of Japan Vol. 185, pp. 9-19, June 1999, (in Japanese).

27) Raven, H., C., "Adequacy of Free-Surface Conditions for the Wave-Resistance Problem", Pro ceedings of 18th Symposium on Naval Hydrodynamics, p. 378, USA, 1990. 